Trauma Berufskrankh 2016 · [Suppl 4]: 18:S391-S394

DOI 10.1007/s10039-016-0122-0

Online publiziert: 8. März 2016

๑) Springer-Verlag Berlin Heidelberg 2016

CrossMark
Peter P. Schmittenbecher

Kinderchirurgische Klinik, Städtisches Klinikum, Karlsruhe, Deutschland

\section{Unterarmschaftfrakturen im Kindesalter}

\section{Konservativ versus operativ}

Die Inzidenz, die operative Therapie und die Komplikationen bei der Unterarmschaftfraktur im Kindesalter haben zugenommen. Nach epidemiologischen Untersuchungen in Finnland werden 4-mal so viele Patienten stationär aufgenommen, die Manipulationen an der oberen Extremität sind um $28 \%$ gestiegen, und der Anteil der Osteosynthesen hat $52 \%$ erreicht. Dabei wird auch in $28 \%$ eine Reintervention fällig, diese gehen jedoch deutlich häufiger (30\%) zulasten der konservativen als der operativen Therapie $(1,4 \%)$. Refrakturen werden in $7,1 \%$, Pseudarthrosen in $1,2 \%$ beobachtet $[1,2]$. All dies zeigt die alltägliche Bedeutung einer zielgerichteten und effektiven Therapie von Unterarmschaftfrakturen im Kindesalter.

\section{Konservative Behandlung}

Die konservative Therapie ist dabei weiterhin indiziert, und nicht jede Anästhesie erfordert zwingend eine Osteosynthese. Lässt sich eine Unterarm- schaftfraktur achsengerecht und stabil reponieren und auch retinieren, spricht nichts gegen die Ruhigstellung im Oberarmgipsverband für 4 Wochen mit einer radiologischen Stellungskontrolle nach 1 Woche, um eine sekundäre Dislokation im Gipsverband auszuschließen respektive darauf reagieren zu können (• Abb. 1). Obwohl es keine Übereinkunft bezüglich der akzeptablen Achsabweichung gibt [3], ist heute hinlänglich bekannt, dass ab einem Achsenfehler von $15^{\circ}$ und je proximaler umso mehr eine Einschränkung der Pronation und/oder Supination zu erwarten ist [4, 5]. Zudem gibt es am Schaft - fernab der Wachstumsfugen - kein relevantes Remodeling ( $\bullet$ Abb. 2), und dies betrifft auch das Kleinkindesalter. Bowman et al. [6] haben an einem zu 90\% konservativ behandelten Patientenkollektiv aufgezeigt, dass bei der Konsolidierung 51\% der Patienten einen Achsenfehler jenseits des Akzeptanzlevels aufwiesen! Über die Hälfte davon waren bereits in der ersten Behandlungswoche aufgetreten und wurden bevorzugt im Alter $>10$ Jahre, bei proximalem Level der Radiusfraktur und bei einer Dislokation der Ulna $>15^{\circ}$ beobachtet. Die oben genannten Kriterien wurden also bestätigt. Yang et al. [7] ergänzten die primär vollständige Fraktur und die unzureichende Reposition als Risikofaktoren für eine Redislokation. Die Grenzen der konservativen Vorgehensweise sind also hinreichend belegt.

\section{Operative Behandlung}

Instabilität ist eine primäre Indikation zur operativen Stabilisierung. Frakturen beider Knochen auf gleicher Höhe mit Quer- oder Schrägfrakturen sind instabil und führen bei konservativer Therapie zu schlechten Ergebnissen. Die elastischstabile intramedulläre Nagelung (ESIN) ist das Verfahren der Wahl und ist bei Erfüllung der Instabilitätskriterien auch im Kleinkindesalter indiziert. Die Implantation der Nägel erfolgt radial retrograd von einem seitlichen oder einem dorsalen Zugang über das Tuberculum

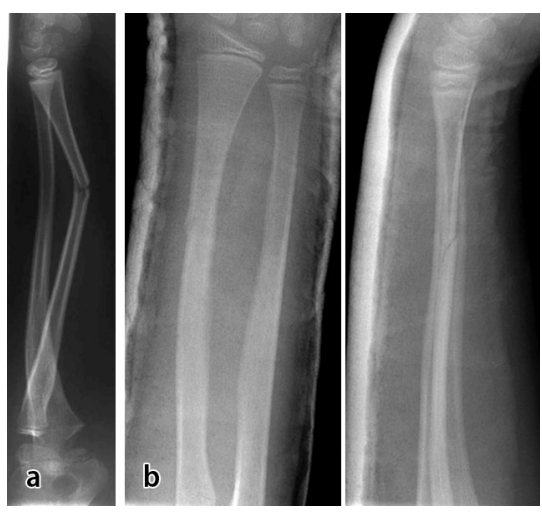

Abb. $1 \Delta$ a Radius-Grünholzfraktur und b achsengerechte Retention im Oberarmgips
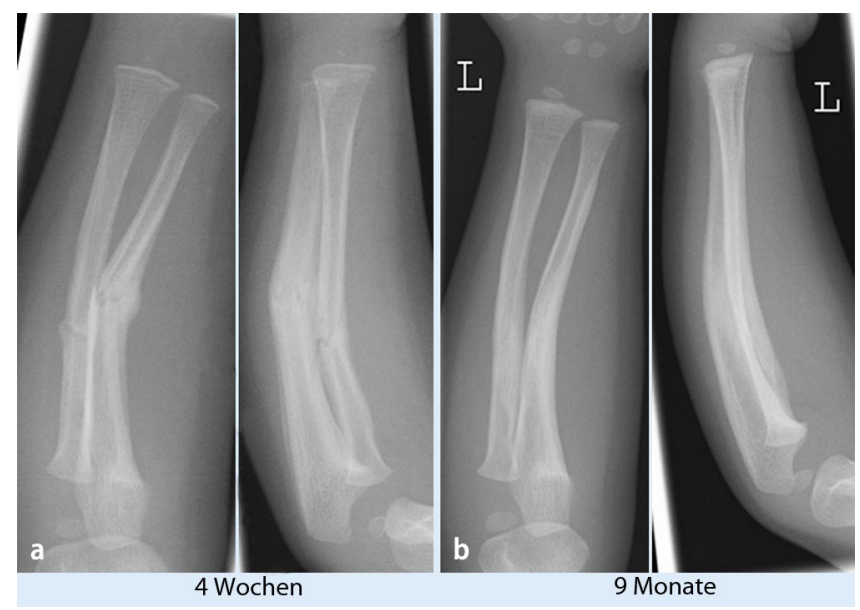

Abb. $2<$ a Fehlheilung einer konservativ behandelten Unterarmschaftfraktur nach 4 Wochen und b unzureichendes Remodeling mit verbleibender Funktionsstörung nach 9 Monaten 

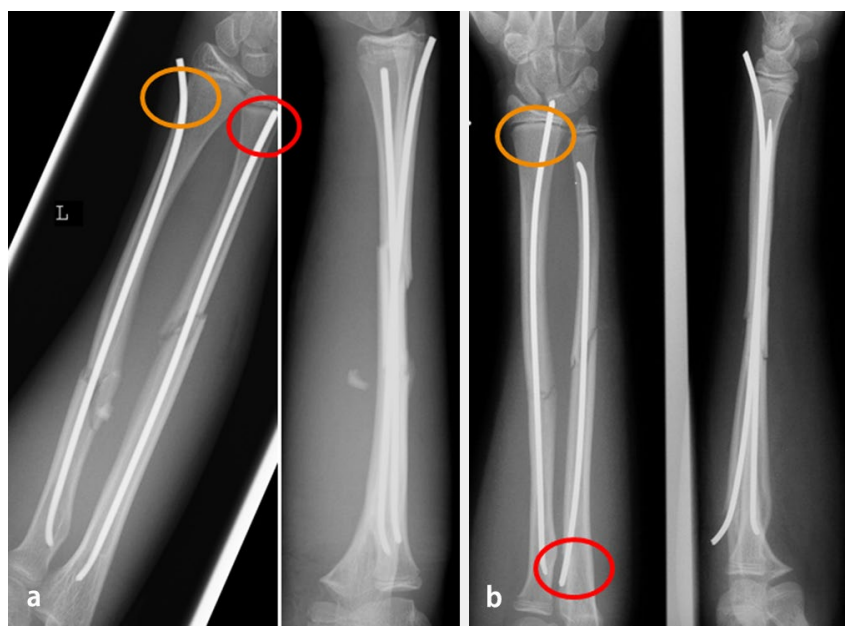

Abb. $3 \Delta$ Mit elastisch-stabiler intramedullärer Nagelung (ESIN) versorgte Unterarmschaftfrakturen mit a retrograder Nagelung beider Knochen von radial lateral und ulnar medial respektive $\mathbf{b}$ gegenläufiger Nagelung von distal radial dorsal und proximal ulnar latera. Orange: radiale Zugänge, rot: ulnare Zugänge
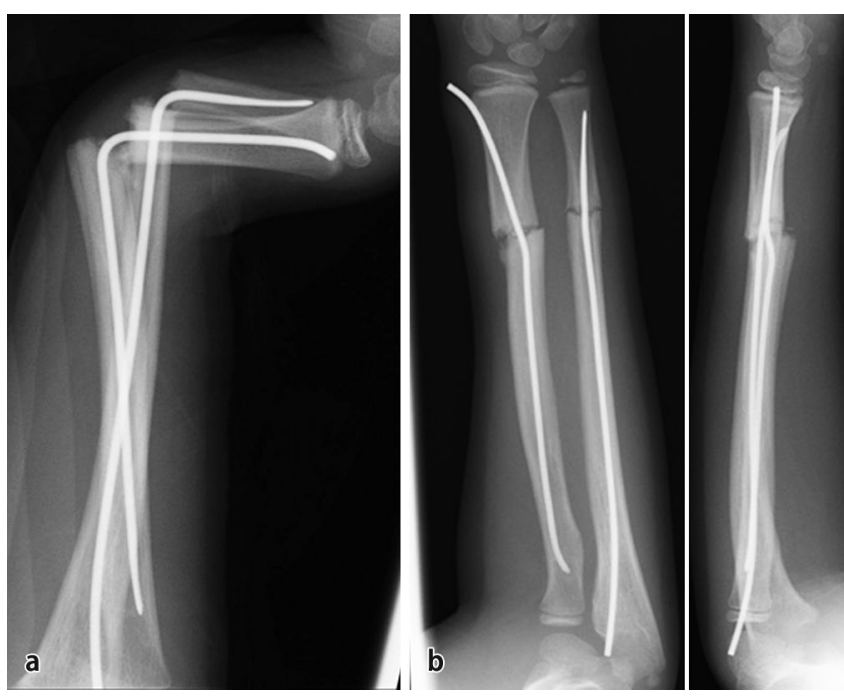

Abb. $4 \Delta$ a Refraktur bei liegenden Nägeln und $\mathbf{b}$ Reposition ohne Nagelwechsel
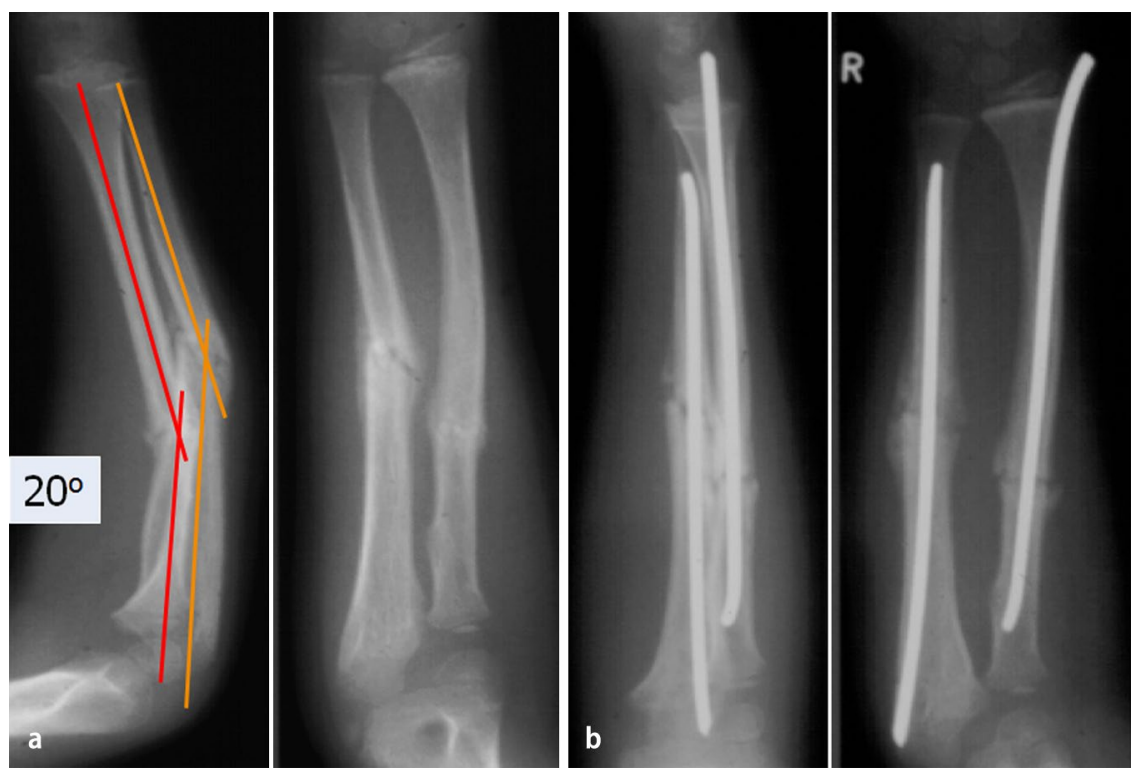

Abb. $5 \Delta$ a Fehlheilung einer konservativ behandelten Unterarmschaftfraktur nach 4 Wochen und $\mathbf{b}$ frühe geschlossene Korrektur mit elastisch-stabiler intramedullärer Nagelung (ESIN)

Listeri aus. Dabei sind lateral der N. radialis superficialis, dorsal die Sehne des M. extensor pollicis longus durch eine ausreichende Inzision und sorgfältige Präparation zu schützen. Eine Stichinzision reicht nicht aus. Die Ulna wird antegrad von der radialen Flanke des Olekranons aus oder ebenfalls retrograd von distal medial genagelt (• Abb. 3). Eine additive Immobilisation ist entbehrlich, die Versorgung ist übungsstabil. Nach 4 Wochen und radiologisch geprüfter ausreichender Kallusbildung erfolgt die Freigabe zum Sport. Die Metallentfernung soll nach vollständiger Durchbauung und abgeschlossenem Remodeling nach 3 bis 6 Monaten geplant werden [8-10]. Kommt es nach der Freigabe bei liegenden Nägeln zur Refraktur, reicht meist die geschlossene Reposition aus (• Abb. 4; [11]), ggf. ist ein Wechsel der Nägel notwendig. Es muss allerdings konstatiert werden, dass es für die Überlegenheit der intramedullären Nagelung über andere Therapieverfahren bis heute keine Evidenz gibt, sodass immer wieder randomisierte Studien angemahnt werden $[12,13]$.

Abwandlungen der intramedullären Nagelung wie sie vornehmlich in USamerikanischen Arbeiten vorgeschlagen werden, dürfen in Anbetracht dessen, dass die biomechanischen Kriterien der elastisch-stabilen 3-Punkt-Verklemmung in keiner Weise erfüllt werden, nicht mit der elastisch-stabilen intramedullären Nagelung (ESIN) verglichen werden. Die Auffädelung der Ulna durch einen transapophysär, gerade eingebrachten Nagel, die Verwendung gerader, nicht verspannter Kirschner-Drähte und die Nagelung nur eines Knochens mit konservativer Behandlung des anderen durch einen zusätzlichen Oberarmgips führen zu Komplikationsraten, die inakzeptabel sind, aber nicht dazu führen dürfen, das Originalverfahren zu diskreditieren [14, 15].

\section{Komplikationen}

Kommt es bei einer konservativen Behandlung nach 4 Wochen zur Konsolidierung in einer funktionell relevanten Fehlstellung, ist die Korrektur dieser Fehlstellung zeitnah am einfachsten. Zeigt das Röntgenbild einen ausreichend weiten Markraum, der nicht durch endostalen Kallus eingeengt ist, ist oftmals die geschlossene Korrektur mittels ESIN möglich (• Abb. 5). Da 
das Wachstum in diesem Bereich kein Remodeling bewirken kann, ist das Abwarten nicht zu empfehlen, denn die Ausrichtung der Membrana interossea in der neuen 3-dimensionalen Konstruktion der Unterarmknochen macht die spätere Korrektur schwieriger und limitiert möglicherweise den funktionalen Erfolg [16].

Komplikationen der ESIN umfassen vornehmlich Weichteilirritationen an den Nagelenden und sind somit vermeidbar [17]. Eine verzögerte Heilung tritt in 1-2\% der Fälle auf und heilt meistens bis 1 Jahr nach dem Unfall ohne ergänzende Maßnahme aus [18, 19]. Geringe Einschränkungen der Umwendbewegungen in den ersten Wochen nach der Osteosynthese sind temporär und auf die oft ausgeprägte Kallusbildung zurückzuführen.

Im Rahmen von Schlichtungsverfahren festgestellte Behandlungsfehler bei der Unterarmschaftfraktur betreffen versäumte Repositionen, versäumte Reaktionen bei sekundärer Dislokation und technische Fehler bei der Osteosynthese [20]. Es ist folglich wichtig, bei der konservativen Behandlung das absolut limitierte Remodeling zu beachten, bei sekundären Zeichen der Instabilität konsequent zu reagieren und bei der operativen Vorgehensweise die technisch einfache und gut reproduzierbare Osteosynthese exakt und unter Einhaltung der biomechanischen Kriterien umzusetzen.

\section{Fazit für die Praxis}

\section{- Unterarmschaftfrakturen sind die häufigsten Schaftfrakturen im Kindesalter. \\ - Neben den konservativen Ver- fahren für stabile Frakturen ge- hört die Osteosynthese mittels ESIN für instabile Frakturen heute ab- solut zum Standard und ist bei technisch korrekter Anwendung als komplikationsarmes und primär definitives Therapieverfahren der ersten Wahl mit sofortiger freier Be- wegung anzusehen.}

Trauma Berufskrankh 2016 · [Suppl 4]: 18:S391-S394 DOI 10.1007/s10039-016-0122-0

(c) Springer-Verlag Berlin Heidelberg 2016

\section{P.P. Schmittenbecher \\ Unterarmschaftfrakturen im Kindesalter.
Konservativ versus operativ}

\section{Zusammenfassung}

Bei steigender Inzidenz der Unterarmschaftfrakturen im Kindesalter ist die konservative Therapie für stabile Frakturen weiterhin empfohlen, sodass Anästhesie nicht zwingend Osteosynthese heißt. Unbefriedigende Verläufe sind je nach Frakturtyp vorauszusehen und radiologisch zu erkennen. Instabile Frakturen erfordern eine Osteosynthese, da die Unterarmschaftfraktur wegen der drohenden Einschränkung der Umwendbewegungen wie eine Gelenkfraktur zu behandeln ist. Die Methodenwahl ist noch ohne Evidenz, jedoch stellt die elastisch-stabile intramedulläre Nagelung (ESIN) heute weltweit das Verfahren der ersten Wahl dar. Sind beide Knochen gebrochen, werden auch beide stabilisiert. Gute Ergebnisse sind allerdings nur bei korrekter Technik zu erwarten. Die Komplikationen sind in der Summe überschaubar und vermeidbar.

Schlüsselwörter

Osteosynthese - Komplikation · Therapie · Anästhesie - Instabilität

\section{Forearm fractures in childhood. Conservative versus operative}

\section{Abstract}

With the increasing incidence of forearm shaft fractures in children, conservative treatment is still recommended for stable fractures, so that anesthesia does not necessarily equate with osteosynthesis. Unsatisfactory courses can be predicted depending on the type of fracture and can be recognized by radiological signs. Unstable fractures require osteosynthesis because they are an imminent danger for limitations of pronation and supination; therefore, they have to be managed in the same way as an articular frac- ture. There is still no evidence for selection of the operative method but elastic stable intramedullary nailing (ESIN) is the method of first choice worldwide. If both bones are broken, both should be stabilized. Good results can be expected only with the correct technique. Complications are in general limited and preventable.

\section{Keywords}

Therapy · Anesthesia · Instability ·

Osteosynthesis · Complications

\section{Korrespondenzadresse

Prof. Dr.P.P.
Schmittenbecher
Kinderchirurgische Klinik,
Städtisches Klinikum
Moltkestr. 90, 76133 Karlsruhe
peter.schmittenbecher@
klinikum-karlsruhe.de

\section{Einhaltung ethischer Richtlinien}

Interessenkonflikt. P.P. Schmittenbecher gibt an dass kein Interessenkonflikt besteht.

Dieser Beitrag beinhaltet keine vom Autor durchgeführten Studien an Menschen oder Tieren.

The supplement containing this article is not sponsored by industry.

\section{Literatur}

1. Helenius I, Lamberg TS, Kääriäinen S, Impinen A, Pakarinen MP (2009) Operative treatment of fractures in children is increasing. J Bone Joint Surg 91:2612-2616

2. Sinikumpu JJ, Lautamo A, Pokka T, Serlo W (2012) The increasing incidence of paediatric diaphyseal both-bone forearm fractures and their internal fixation during the last decade. Injury 43:362-366

3. Ploegmakers JW, Verheyen CCPM (2006) Acceptance of angulation in the non-operative treatment of paediatric forearm fractures. J Pediatr Orthop B 15:428-432

4. Colaris J, Allema JH, Reijman M, de Vries MR, Biter UL, Bloem RM, van de Ven CP, Verhaar JA (2014) Which factors affect limitation of pronation/ supination after forearm fractures in children? A prospective multicenter study. Injury 45:696-700

5. Weinberg A-M, Kasten P, Castellani C et al (2001) Which axial deviation results in limitations of proand supination following diaphyseal lower arm fractures in children. Eur J Trauma 27:309-316 
6. Bowman EN, Mehlman CT, Lindsell CJ, Tamai J (2011) Nonoperative treatment of both-bone forearm shaft fractures in children: predictors of early radiographic failure. J Pediatr Orthop 31:2332

7. Yang JJ, Chang JH, Lin KY, Lin LC, Kuo CL (2012) Redisplacement of diaphyseal fractures oft he forearm after closed reduction in children: a retrospective analysis of risk factors. J Orthop Trauma 26:110-116

8. Dietz HG, Schmittenbecher PP, Slongo T, Wilkins KE (2006) Elastic stable intramedullary nailing (ESIN) in children - AO Manual of fracture management. Thieme, Stuttgart, S 71-107

9. Lieber J, Joeris A, Knorr P, Schalamon J, Schmittenbecher PP (2005) ESIN in forearm fractures. Eur J Trauma 31:3-11

10. Schmittenbecher PP (2005) State-of-the-art treatment of forearm shaft fractures. Injury 36(Suppl 1):A25-A34

11. van Egmond $P W$, van der Sluijs $H A$, van Royen BJ, Saouti R (2013) Refractures of the paediatric forearm with the intramedullary nail in situ. $\mathrm{Br}$ Med J Case Rep 24:2013

12. Abraham A, Kumar S, Chaudry S, Ibrahim T (2011) Surgical interventions for diaphyseal fractures of the radius and ulna in children. Cochrane Database Syst Rev (11):CD007907. doi:10.1002/14651858. CD007907.pub2

13. Franklin CC, Robinson J, Noonan K, Flynn JM (2012) Evidence-based medicine: management of pediatric forearm fractures. J Pediatr Orthop 32(Suppl 2):S131-S134

14. Dietz JF, Bae DS, Reiff E, Zurakowski D, Water PM (2010) Single bone intramedullary fixation of the ulna in pediatric both bone forearm fractures: analysis of short-term clinical and radiographic results. J Pediatr Orthop 30:420-424

15. Flynn JM, Jones KJ, Garner MR, Goebel J (2010) Eleven years experience in the operative management of pediatric forearm fractures. J Pediatr Orthop 30:313-319

16. Schmittenbecher PP (2012) Posttraumatische (Wachstums-) Störungen am Unterarm im Kindesalter. Trauma Berufskrankh 14:255-262

17. Parikh SN, Jain VV, Denning J, Tamai J, Mehlman CT, McCarthy JJ, Wall EJ, Crawford AH (2012) Complications of elastic stable intramedullary nailing in pediatric fracture management: AAOS exhibit selection. J Bone Joint Surg 94:e184

18. Fernandez FF, Eberhardt O, Langendörfer M, Wirth $T$ (2009) Nonunion of forearm shaft fractures in children after intramedullary nailing. J Pediatr Orthop B 20:1473-5865

19. Schmittenbecher PP, Fitze G, Gödecke J et al (2008) Delayed healing of forearm shaft fractures in children after intramedullary nailing. J Pediatr Orthop 28:303-306

20. Vinz H, Festge OA, Neu J (2012) Malpractice in the treatment of diaphyseal fractures in children - experience of the Arbitration Office of the Northern German Medical Boards. Z Orthop Unfall 150:648-656 